\title{
Significance of Serum Uric Acid in Children with Pulmonary Arterial Hypertension
}

\author{
Carmen Corina Şuteu' ${ }^{1}$ Theodora Benedek², Rodica Togănel ${ }^{1}$ \\ 1 Department M4, IIIrd Pediatric Discipline, University of Medicine and Pharmacy, Tîrgu Mureş, Romania \\ 2 Department M3, Clinic of Cardiology, University of Medicine and Pharmacy, Tîrgu Mureş, Romania
}

\section{CORRESPONDENCE \\ Carmen Corina Șuteu \\ 38 Gheorghe Marinescu St \\ 540139 Tîrgu Mureș, Romania \\ Tel: 0265215551 \\ Email: suteucarmen@yahoo.com}

\section{ARTICLE HISTORY}

Received: 1 April, 2016

Accepted: 15 May, 2016
Theodora Benedek • 38 Gheorghe Marinescu St, 540139 Tîrgu Mureș, Romania. Tel: +40 265215551 Email: theodora.benedek@gmail.com

Rodica Togănel • 38 Gheorghe Marinescu St, 540139 Tîrgu Mureș, Romania. Tel: +40 265215551

Email: rodicatoganel@yahoo.com

\begin{abstract}
Introduction: Pulmonary arterial hypertension (PAH) is a complex disease with poor prognosis. Serum uric acid has been proposed as a potentially non-invasive and objective parameter for prognosis and response to therapy. Objectives: To investigate the potent relationship between serum uric acid levels and functional and echocardiographic parameters in children with PAH. Methods: Serum uric acid levels were measured in 34 children with PAH and were correlated with the functional class, 6-minute walk test, and echocardiographic parameters at baseline and at 12 months follow-up. Results: In pediatric PAH patients serum uric acid levels were higher compared with the control subjects $(p=0.001)$. In the high uric acid group serum uric acid levels were correlated with 6 -minute walk test $(p=0.008)$, and with several echocardiographic parameters, such as pulmonary vascular resistance $(p=0.04)$, fractional area change $(p=0.05)$, left ventricle eccentricity index $(p=0.04)$, right atrial area $(p=0.03)$, right ventricle myocardial index $(p=0.01)$, and pericardial effusion $(p=0.001)$, markers of right ventricular overload and dysfunction. Conclusions: Serum uric acid levels are easy to collect and measure, and correlate with both functional and echocardiographic parameters that reflect right ventricular dysfunction.
\end{abstract}

Keywords: uric acid, pulmonary arterial hypertension, children

\section{BACKGROUND}

Pulmonary arterial hypertension $(\mathrm{PAH})$ is a rare, progressive disease, characterized by an increase in pulmonary vascular resistance (PVR) due to vasoconstriction and remodeling, leading to increased pulmonary arterial pressure (PAP), right ventricular (RV) failure, and ultimately, death. ${ }^{1}$ Serum uric acid (UA), the final oxidation product of the purinic metabolism, acts as an endogenous free radical scavenger, protecting cells from the damage caused by reactive oxygen species and reactive nitrogen species. ${ }^{1}$ Several studies have demonstrated that UA serum levels are increased in conditions of impaired oxidative metabolism such as congestive heart failure, ${ }^{2}$ pulmonary embolism, ${ }^{3}$ primary pulmonary hypertension (IPAH), ${ }^{4}$ Eisenmenger syndrome, ${ }^{5}$ and PAH due to systemic sclerosis. ${ }^{6}$ Uric acid levels are elevated and correlated with the severity of disease in PAH. Nagaya et al., in a study of 99 IPAH patients and age-matched controls, 
showed that elevated serum UA levels were correlated with pulmonary haemodynamics, that it had a strong association with long-term mortality, and that it had decreased with vasodilator therapy. ${ }^{4}$

Several mechanisms may be involved in the presence of high UA levels in PAH, but the exact metabolic mechanism is not clear. Lung tissue ischemia, impaired renal perfusion and urinary excretion of UA may be factors responsible for the elevation of UA levels in patients with IPAH.7 Recent evidence suggests that UA inhibits acetylcholine-mediated vasodilatation by acting on the vascular endothelium. ${ }^{8}$

There is very little information available on the clinical significance of UA in pediatric patients with PAH. We hypothesized that UA levels are elevated in pediatric patients with $\mathrm{PAH}$ and are associated with functional and echocardiographic parameters, thus having a prognostic value in these populations.

\section{METHODS}

Patients selection. The present study was conducted in accordance with the Declaration of Helsinki and was approved by the local ethics committee. Informed consent was obtained from the parents of all children included in the study.

A total of 34 pediatric patients with $\mathrm{PAH}$ and 34 controls were evaluated, between December 2013 and January 2015 in this prospective, non-interventional study. The pediatric patients with $\mathrm{PAH}$ were classified in clinical forms of PAH: IPAH and PAH associated with congenital heart disease (CHD).

Diagnosis of PAH. PAH was defined in accordance with ESC/ERS Guidelines as an increase in the mean pulmonary arterial pressure $\geq 25 \mathrm{mmHg}$ at rest as assessed by right heart catheterization (RHC), a pulmonary artery wedge pressure (PAWP) $\leq 15 \mathrm{mmHg}$, and a PVR $>3$ Wood units in the absence of other causes of pre-capillary $\mathrm{PH}$ such as $\mathrm{PH}$ due to lung diseases, chronic thromboembolic $\mathrm{PH}$ or other rare diseases. ${ }^{9}$ In all cases, cardiac catheterization was performed at presentation to confirm the diagnosis of PAH. Patients were treated with specific vasodilator therapy in accordance with the guideline recommendations.

Functional capacity. Baseline and follow-up clinical assessment included World Health Organization functional class (WHO FC) and exercise capacity assessed by 6-minute walk test (6MWT) distance (in children $\geq 5$ years old).

Transthoracic echocardiography. All patients underwent echocardiographic evaluation using a IE33 Philips ECHO device, according to the previously described protocol. ${ }^{10}$ The parameters that reflect the RV function: tricuspid annular plane systolic excursion (TAPSE), tricus- pid annular velocity (S'), fractional area change (FAC), myocardial performance index (Tei), pericardial effusion, the pulsed wave Doppler interogation of the trans-tricuspid inflow, tissue Doppler, E/E` ratio; the parameters that reflect the RV anatomy and physiology: RA area, tricuspid annulus diameter, pulmonary artery annulus diameter, left ventricle eccentricity index (LVEI); and the parameters that reflect the hemodynamics: systolic pulmonary artery pressure (sPAP), mean pulmonary artery pressure (mPAP), acceleration time (ACT), pulmonary vascular resistance (PVR) - were evaluated by echocardiography. For estimation of the PVR we used an equation using the ratio of sPAP to RV outflow tract velocity time integral (RVOT VTI) and a constant designating presence of RVOT VTI midsystolic notching. ${ }^{11}$

Measurement of serum UA levels. Venous blood was obtained for the measurement of serum UA levels at baseline, and functional and echocardiographic assessment was also performed. Serum UA levels (range $3.5-7.2 \mathrm{mg} / \mathrm{dl}$ ) were determined using the uricase-peroxidase method. In addition, serum levels of urea, creatinine, aspartate transaminase (AST), alanine transaminase (ALT), gamma-glutamyl transferase (gamma-GT), bilirubin and sodium (Na) were measured in each patient in close temporal proximity to the echocardiographic study.

Patient follow-up. In pediatric patients with $\mathrm{PAH}$ the serum UA levels were correlated with WHO FC, exercise capacity estimated by the $6 \mathrm{MWT}$, and echocardiographic parameters with prognostic value at initiation of the study and 12 months later.

\section{RESULTS}

A total of 68 subjects, 34 (50\%) pediatric patients with $\mathrm{PAH}$ and 34 (50\%) age-matched controls, were included in the study. In the PAH group, $55.8 \%(\mathrm{n}=19)$ were female, the mean age was 9.13 years and the average weight was 27.01 $\mathrm{kg}$. The different etiologies of PAH included: IPAH $(\mathrm{n}=5$, $14.7 \%)$ and PAH associated with CHD ( $\mathrm{n}=29,85.29 \%)$. WHO FC II, III and IV were recorded in $35.29 \%(\mathrm{n}=12)$, $61.76 \%(n=21)$, and $2.94 \%(n=1)$ of cases, respectively.

Serum UA levels. Serum UA levels were significantly higher in patients with PAH compared with the control group $(\mathrm{p}=0.001)$. The pediatric patients with PAH were grouped according to the mean value of serum UA $(5 \mathrm{mg} /$ $\mathrm{dl}$ ), and the results are summarized in Table 1 . There were no significant differences between the high UA group and the low UA group regarding age, sex, and weight. Initial UA levels did not differ between patients with idiopathic or secondary PAH. Serum urea $(p=0.05)$ and creatinine 
TABLE 1. Comparison of patient characteristics according to median value of serum uric acid levels

\begin{tabular}{|c|c|c|c|}
\hline Variables & $\begin{array}{l}\text { Low UA group } \\
\quad(n=17)\end{array}$ & $\begin{array}{l}\text { High UA group } \\
\quad(n=17)\end{array}$ & $p$ value \\
\hline Age, yr & $8.94 \pm 2.67$ & $9.33 \pm 3.43$ & 0.78 \\
\hline Female gender & $11(64.7 \%)$ & 8 (47.05\%) & 0.76 \\
\hline Weight, kg & $23.3 \pm 9.6$ & $30.7 \pm 12.78$ & 0.06 \\
\hline \multicolumn{4}{|l|}{$\mathrm{PAH}$} \\
\hline IPAH & $2(11.7 \%)$ & 3 (17.6\%) & 0.54 \\
\hline $\mathrm{CHD}-\mathrm{PAH}$ & 15 (29.39\%) & $14(27.41 \%)$ & 0.23 \\
\hline $\mathrm{FC}\|/\| / 1 \mathrm{IV}$ & $8 / 9$ & $4 / 12 / 1$ & 0.32 \\
\hline 6MWT distance, m & $437.4 \pm 67.3$ & $303.1 \pm 114.9$ & 0.008 \\
\hline \multicolumn{4}{|l|}{ Laboratory findings } \\
\hline Urea, mg/dL & $27.16 \pm 8.19$ & $33.7 \pm 10.6$ & 0.05 \\
\hline Creatinine, mg/dL & $0.47 \pm 0.11$ & $0.60 \pm 0.11$ & 0.002 \\
\hline AST, U/L & $22.4(11-50)$ & $24.9(13.3-61.8)$ & 0.40 \\
\hline $\mathrm{ALT}, \mathrm{U} / \mathrm{L}$ & $14.1(8.7-26)$ & $14.1(6.6-162)$ & 0.54 \\
\hline gamma-GT, U/L & $15(9-52)$ & $20(7-69)$ & 0.14 \\
\hline Bilirubin, mg/dL & $0.48 \pm 0.13$ & $0.71 \pm 0.26$ & 0.07 \\
\hline $\mathrm{Na}, \mathrm{mmol} / \mathrm{L}$ & $139.9 \pm 1.85$ & $139.7 \pm 4.02$ & 0.84 \\
\hline \multicolumn{4}{|l|}{ Echocardiography } \\
\hline SPAP & $92.69 \pm 28.89$ & $107.7 \pm 18.8$ & 0.11 \\
\hline mPAP & $56.2 \pm 18.1$ & $66.7 \pm 13.5$ & 0.12 \\
\hline ACT & $73.4 \pm 12.9$ & $67.01 \pm 12.1$ & 0.22 \\
\hline PVR & $8.7 \pm 2.37$ & $12.4 \pm 6.06$ & 0.04 \\
\hline LVEI & $1.26 \pm 0.24$ & $1.49 \pm 0.34$ & 0.04 \\
\hline RA area & $11.9 \pm 4.36$ & $20.4 \pm 4.2$ & 0.03 \\
\hline Tr diameter & $23.8 \pm 4.41$ & $28.4 \pm 6.22$ & 0.03 \\
\hline PA diameter & $21.8 \pm 5.24$ & $22.7 \pm 4.06$ & 0.67 \\
\hline TAPSE & $17.5 \pm 4.38$ & $20.0 \pm 6.77$ & 0.35 \\
\hline FAC & $39.7 \pm 7.12$ & $32.7 \pm 11.4$ & 0.05 \\
\hline Tei & $0.46 \pm 0.23$ & $0.470 \pm 0.24$ & 0.01 \\
\hline Pericardial effusion & 0 & $35.3 \%$ & 0.001 \\
\hline$E / A$ & $1.17 \pm 0.38$ & $1.28 \pm 0.60$ & 0.57 \\
\hline$S^{\prime}$ & $10.2 \pm 2.38$ & $8.97 \pm 3.38$ & 0.25 \\
\hline$E / E^{\prime}$ & $0.0 .5 \pm 0.02$ & $0.06 \pm 0.01$ & 0.44 \\
\hline
\end{tabular}

( $\mathrm{p}=0.002)$ levels were also higher in the high UA group.

Serum UA levels and functional parameters. Initially we could not prove a statistically significant correlation between UA and WHO FC ( $\mathrm{p}=0.17)$, and we did not observe a correlation between serum levels of UA and FC during follow-up. A higher WHO FC was associated with a decreased 6MWT. Serum UA level $(\mathrm{p}=0.0001, \mathrm{r}=$ $-0.8284)$ negatively correlated with $6 \mathrm{MWT}$ as tested on the day of the blood sampling (Figure 1). The 6MWT distance was significantly lower in the high UA group than in the low UA group ( $\mathrm{p}=0.008)$.

Serum UA levels and echocardiographic parameters. High UA levels were positively correlated with echocardiographic variables, which illustrated: the degree of RV overload, such as PVR $(p=0.04)$, and the RV function, such as FAC $(p=0.05)$, Tei $(p=0.01)$ and pericardial effu- sion $(\mathrm{p}=0.001)$, and with echo parameters that reflect $\mathrm{RV}$ anatomy, such as LVEI $(\mathrm{p}=0.04)$, RA area $(\mathrm{p}=0.03)$ and Tr diameter $(\mathrm{p}=0.03)$.

Prognosis value of serum UA levels. The pediatric PAH patients included in the study were treated with specific pulmonary vasodilator therapy. At the time of inclusion, 18 patients received Bosentan, 9 patients received Sildenafil and 7 patients received combined therapy. In the case of 3 patients ( 2 patients with IPAH and 1 patient with $\mathrm{PAH}$ associated with CHD), during follow-up, we found a worsening of the functional class, and, in addition, the echocardiographic parameters indicated a more compromised RV function, requiring intensification of the specific therapy.

High UA levels predicted the progression of right ventricular failure. ROC-curves for UA (area under the curve 


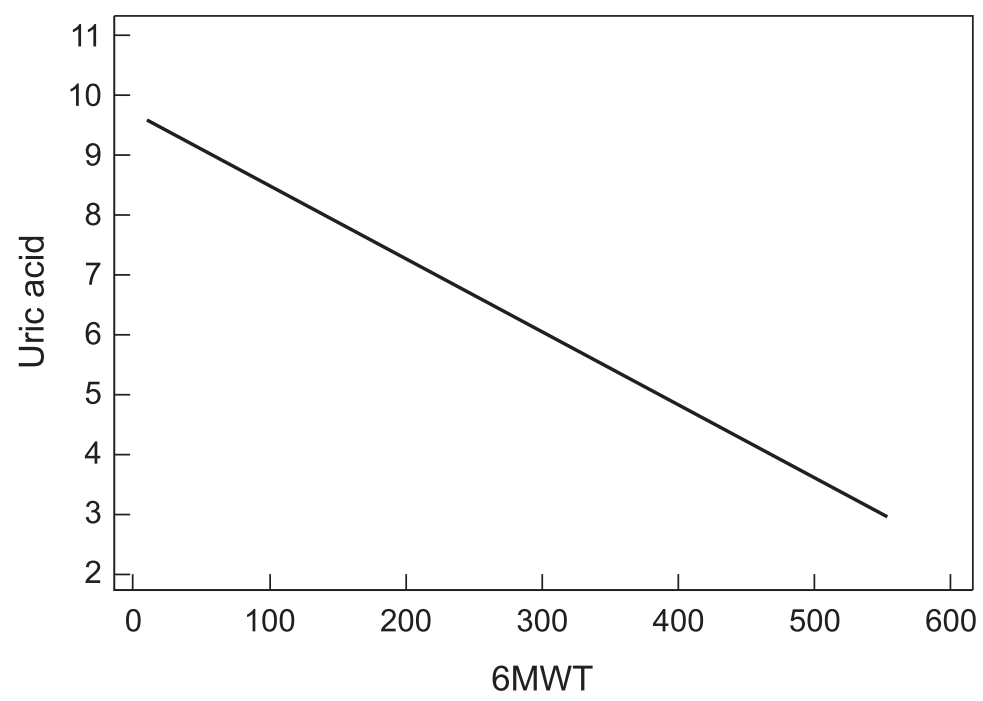

FIGURE 1. Relation between serum uric acid and 6-minute walk test in pediatric patients with $\mathrm{PAH}$ at baseline $(r=-0.82, \mathrm{p}<0.0001)$

AUC $>0.80$ ) predicting the RV dysfunction are provided in Figure 2. An initial serum UA cutoff value of $8.53 \mathrm{pg} / \mathrm{ml}$ for prediction of progression of the RV dysfunction was identified by ROC analysis (AUC $=0.86$ (95\% CI: 0.698-0.955)). When using this cutoff value, the test showed to have a $66.67 \%$ sensitivity (95\% CI: $9.4-99.2$ ), and a $100 \%$ specificity (95\% CI: 88.8-100) in predicting a negative outcome.

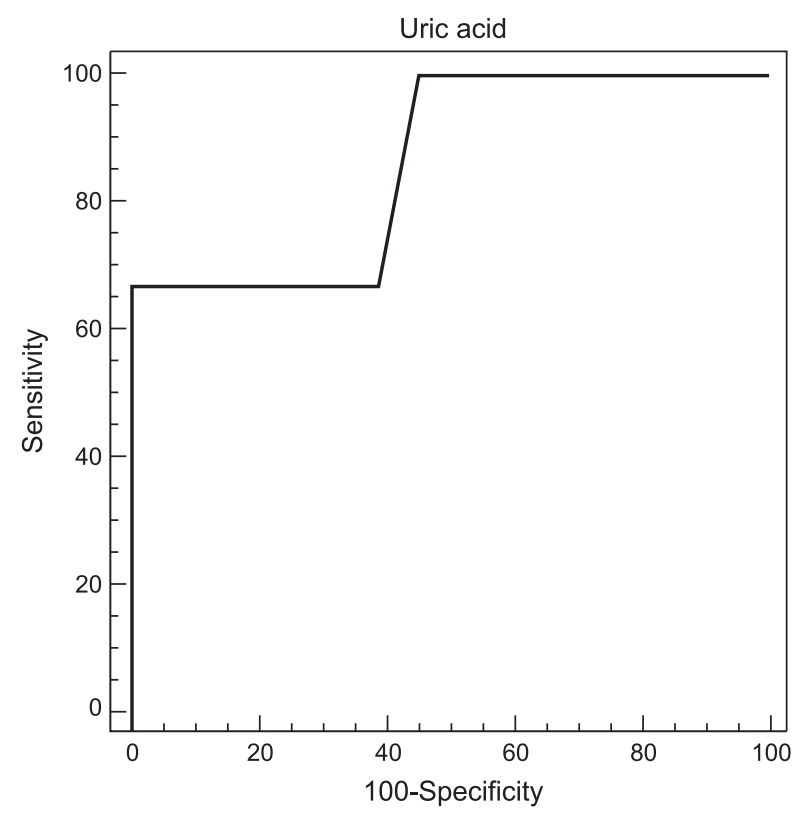

FIGURE 2. ROC analysis for serum uric acid ( $A \cup C=0.86,95 \% \mathrm{Cl}$ $0.698-0.955, p=0.01$ ) in the prediction of the progression of the right heart failure in pediatric patients with $\mathrm{PAH}$

\section{DISCUSSIONS}

In this study, we observed that the serum levels of UA in pediatic patients with $\mathrm{PAH}$ were higher than in the healthy subjects. There was a positive correlation between UA levels and exercise capacity assessed by $6 \mathrm{MWT}$, and with some ecocardiogrphic parameters with pronostic value. In addition, our study indicated that an elevated serum UA level at baseline was relevant to long-term outcome for pediatric patients with PAH.

Our results demonstrated that pediatric patients with elevated serum UA ( $>5 \mathrm{mg} / \mathrm{dl}$ ) levels showed increased severity according to the 6MWT, which is a marker of severity in $\mathrm{PAH}$ and a predictor for the outcome of PAH patients. A positive correlation between serum UA levels and the functional parameters has also been observed in adult patients with PAH. ${ }^{6}$ In a study involving 29 patients with $\mathrm{PAH}$, UA levels correlated positively with FC and negatively with $6 \mathrm{MWT}$ scores. ${ }^{12}$

Van Albada et al. investigated the correlations between several serum markers in children with $\mathrm{PAH}$, and they found that UA serum levels correlated with invasively obtained hemodynamic data. ${ }^{13}$ In an adult population, Voelkel A et al. demonstrated that there is a positive correlation between right atrial pressure elevation and serum UA levels. ${ }^{14}$ We did not demonstrate the correlation of serum biomarkers with hemodynamic data because the RHC was not performed in this study, but all patients included in the study were evaluated by echocardiography, which is the standard method for non-invasive monitoring of PAH progression. ${ }^{15,16}$ Echocardiographic findings reli- 
ably provide several estimates for hemodynamic functions that closely correlate with the measurements obtained by RHC. ${ }^{17-22}$ We could demonstrate a statistically significant positive correlation between high serum UA levels and echocardiographic parameters of RV impairment which include RA area $(\mathrm{p}=0.03)$, the LVEI $(\mathrm{p}=0.04)$, Tei $(\mathrm{p}=$ $0.01)$, FAC $(\mathrm{p}=0.05)$ and pericardial effusion $(\mathrm{p}=0.001)$. Identifying an elevated PVR is critical for initiating appropriate treatment. Recently, Opotowsky AR et al., in a study that included $217 \mathrm{PAH}$ patients, presented an equation that estimates the PVR by echocardiography using the ratio of sPAP to RVOT VTI and a constant designating presence of RVOT VTI mid-systolic notching. ${ }^{11}$ Because RHC is an invasive method that requires general anesthesia in children, we could not apply it repeatedly. For monitoring response to therapy, we estimated the PVR by using the equation proposed by Opotowski. Our study revealed statistically positive correlations between high serum UA levels and PVR estimated by echocardiography $(\mathrm{p}=0.04)$. This study demonstrated that serum levels of UA may provide an idea regarding right cardiac echo parameters.

Zhang $\mathrm{C}$ et al., in a study on 86 adult IPAH patients and age-matched controls, concluded that the levels of serum UA were related to the severity of PAH and the ventricular dysfunction assessed by trans-thoracic echocardiography. ${ }^{7}$

Uric acid level has been described as a prognostic factor for adults with PAH.4,10,12 In the current pediatric series, the ROC-curve analysis showed that an UA level $>8.53$ $\mathrm{mg} / \mathrm{dl}$ can be used for PAH progression assessment in pediatric patients with $\mathrm{PAH}$.

The limits of this study were the relatively small number of pediatric patients diagnosed with PAH and the heterogeneity of the studied group. In addition, all patients included in this study survived, so the ability of serum UA level to predict mortality was not assessed. More thorough investigations of the predictive ability of a high UA value are needed.

\section{CONCLUSIONS}

Our data indicate that high serum UA levels correlate with both functional and echocardiographic parameters that reflect RV dysfunction. The present results indicate that serum UA levels could be easy to collect and measure, and it may provide a reproducible biological marker of unfavorable outcomes in pediatric patients with severe PAH.

\section{CONFLICT OF INTEREST}

None declared.

\section{ACKNOWLEDGEMENT}

This paper is supported by the Sectoral Operational Programme Human Resources Development (SOP HRD), financed from the European Social Fund and by the Romanian Government under the contract numberPOSDRU/159/1.5/S/137390.

\section{REFERENCES}

1. Pezzuto B, Badagliacca R, Poscia R, et al. Circulating biomarkers in pulmonary arterial hypertension: Update and future direction. J Heart Lung Transplant. 2015;34:282-305.

2. Anker SD, Doehner W, Rauchhaus $M$, et al. Uric acid and survival in chronic heart failure: validation and application in metabolic, functional, and hemodynamic staging. Circulation. 2003;107:1991-1997.

3. Shimizu Y, Nagaya N, Satoh T, et al. Serum acid uric level increases in proportion to the severity of pulmonary thromboembolism. Circ J. 2002;66:571-575.

4. Nagaya N, Uematsu M, Satoh T, et al. Serum uric acid levels correlate with the severity and the mortality of primary pulmonary hypertension. Am J Respir Crit Care Med. 1999;160:487-492.

5. Oya H, Nagaya N, Satoh T, et al. Haynamic correlates and pronostic significance of uric acid in adults patients with Eisenmenger syndrome. Heart. 2000;84:53-58

6. Dimitroulas T, Giannakoulas G, Dimitroula H, et al. Significance of serum uric acid in pulmonary hypertension dueto systemic slerosis: a pilot study. Rheumatol Int. 2011;31:263-267.

7. Zang CY, Ma LL, Wang CX. Relationship between serum uric acid levels and ventricular function in patients with idiopathic pulmonary hypertension. Exp Clin Cardiol. 2013;18(1):37-39

8. Khosla UM, Zharikov S, Finch JL, et al. Hyperuricemia induces endothelium dysfunction. Kidney Int. 2005;67:1739-1742.

9. Galie N, Humbert M, Vachiery JL, et al. 2015 ESC/ERS Guidelines for the diagnosis and treatment of pulmonary hypertension. Eur Heart $\mathrm{J}$. 2016;37(1):67-119

10. Suteu C, Blesneac C, Toganel R, et al. Epidemiological characteristics and prospective follow-up of children with pulmonary arterial hypertension. Acta Medica Marisiensis. 2015;61(2):75-82.

11. Opotowsky AR, Clair M, Afilalo J. A simple echocardiographic method to estimate pulmonary vascular resistance. Am J Cardiol. 2013;112:873-882.

12. Bendayan D, Shitrit D, Ygla M, et al. Hyperuricemia as a pronostic factor in pulmonary arterial hypertension. Respir Med. 2003;97:130-133.

13. Van Albada ME, Loot FG, Fokkema R, et al. Biological serum markers in the management of pediatric pulmonary arterial hypertension. Pediatric Research. 2008;63(3):321-327.

14. Voelkel MA, Wynne KM, Badesch DB, et al. Hyperuricemia in severe pulmonary hypertension. Chest. 2000;117:19-24.

15. Bossome E, Bodini BD, Mazza A, et al. Pulmonary arterial hypertension, the key role of echocardiography. Chest. 2005;127:1836-1843.

16. Galie N, Hinderliter AL, Torbicki A, et al. Effects of the oral endothelinreceptor antagonist bosentan on echocardiographic and Doppler measures in patients with pulmonary arterial hypertension. J Am Coll Cardiol. 2003;41:1380-1386

17. Colvin KL, Dufva MJ, Delaney RP, et al. Biomarkers for pediatric pulmonary arterial hypertension- a call to collaborate. Frontiers in Pediatrics. 2014;2(7):1-12

18. Raymond RJ, Hinderliter AL, Willis PW, et al. Echocardiographic predictors of adverse outcomes in primary pulmonary hypertension. J Am Coll Cardiol. 2002;39:1214-1219.

19. Eysmann SB, Palevsky HI, Reichek N, et al. Two-dimensional and Doppler echocardiography and cardiac catheterisation correlates of survival in primary pulmonary hypertension. Circulation. 1989;80:353-360.

20. Vonk MC, Sander MH, van der Hoogen, et al. Right ventricle Tei-index: a tool to increase the accuracy of non-invasive detection of pulmonary arterial hypertension in connective tissue disease. Eur J Echocardiogr. 2007;8:317-321.

21. Anavekar NS, Gerson D, Skali H, et al. Two- dimensional assessment of right ventricular function: an echocardiographic-MRI correlative study. Echocardiography. 2007;24(5):452-456.

22. Jone PN, Ivy DD. Echocardiography in pediatric pulmonary hypertension. Frontiers in paediatrics. 2014;124(2). 